\title{
Optimization based on electro-thermo-mechanical modeling of the high electron mobility transistor (HEMT)
}

\author{
Abdelhamid Amar ${ }^{1,2, *}$, Bouchaïb Radi ${ }^{2}$, and Abdelkhalak El Hami ${ }^{1}$ \\ 1 Normandie University, INSA Rouen, LMN, 76000 Rouen, France \\ ${ }^{2}$ Hassan First University of Settat, FST, LIMII, BP: 577, Route de Casa, Settat, Morocco
}

Received: 30 December 2020 / Accepted: 27 October 2021

\begin{abstract}
The electro-thermomechanical modeling study of the High Electron Mobility Transistor (HEMT) has been presented, all the necessary equations are detailed and coupled. This proposed modeling by the finite element method using the Comsol multiphysics software, allowed to study the multiphysics behaviour of the transistor and to observe the different degradations in the structure of the component. Then, an optimization study is necessary to avoid failures in the transistor. In this work, we have used the Covariance Matrix Adaptation-Evolution Strategy (CMA-ES) method to solve the optimization problem, but it requires a very important computing time. Therefore, we proposed the kriging assisted CMA-ES method (KA-CMA-ES), it is an integration of the kriging metamodel in the CMA-ES method, it allows us to solve the problem of optimization and overcome the constraint of calculation time. All these methods are well detailed in this paper. The coupling of the finite element model developed on Comsol Multiphysics and the KA-CMA-ES method on Matlab software, allowed to optimize the multiphysics behaviour of the transistors. We made a comparison between the results of the numerical simulations of the initial state and the optimal state of the component. It was found that the proposed KA-CMA-ES method is efficient in solving optimization problems.
\end{abstract}

Keywords: HEMT / electro-thermomechanical / optimization / KA-CMA-ES

\section{Introduction}

The transistor is a very important electronic component in mechatronic systems, it ensures several functions such as control and signal amplification. The high electron mobility transistor (HEMT) based on aluminium-gallium nitride/gallium nitride ( $\mathrm{AlGaN} / \mathrm{GaN}$ ), is a very important electronic component due to its structure and materials. Gallium nitride (GaN) is characterized by high mobility, a very high electric breakdown field and high thermal conductivity which is a great advantage compared to other types of transistor [1,2]. Thanks to these and other characteristics, these transistors are used in several highfrequency and high-temperature applications [3,4], such as, telecommunication, electronic warfare (military field) and airborne systems $[5,6]$, etc. HEMT has also been used in several systems such as high power amplifiers, radars and satellites, it is also found in radio frequency sensors and devices [7]. The operating temperature is a very important factor that can influence the HEMT reliability, because most of its characteristics such as electron mobility,

\footnotetext{
* e-mail: abdelhamid.amar@insa-rouen.fr
}

thermal conductivity and saturation rate are temperaturedependent [8], they can be influenced by the operating temperature $[9,10]$. All these characteristics tend to degrade due to the phenomenon of transistor self-heating $[11,12]$. In addition, the operating conditions influence the mechanical behaviour of the HEMT structure, these conditions generate displacements, strains and stresses in the structure [13,14]. These electro-thermomechanical phenomena give rise to degradations such as burying the gate, damaging the connection between the chip and the package [4], degradation of electron mobility and current reduction [15]. In the case of airborne systems, the majority of failures are caused by high-power amplifiers at its highpower transistors. These failures are due to the operating conditions of the transistors, which negatively influence their reliability and therefore that of the systems [16]. Because of all these constraints, it is necessary to start a modeling study to understand the HEMT multiphysics behaviour and to optimize its reliability. Among the optimization methods, there is the CMA-ES (Covariance Matrix Adaptation-Evolution Strategy) method. It's a stochastic research method based on the population in continuous and discrete spaces [17], it is used to solve optimization problems by minimizing an objective 
function, the objective function to be minimized depends on the failure mode. This method has shown its efficiency through results obtained in several works which aim at the reliability of systems [18]. The CMA-ES method is based on the finite element model to solve the optimization problem but, this method is no longer practicable when the finite element model takes tens of minutes. Whereas, the optimization process requires hundreds or thousands of simulations. By integrating the CMA-ES method with the kriging metamodel, we obtain the KA-CMA-ES method (Krigin assisted-Covariance Matrix Adaptation-Evolution Strategy) which allows overcoming the calculation time constraint.

This paper is organized as follows. Firstly, a description of the HEMT, its structure and its operating mode will be given. Then we will develop an electro-thermomechanical model, it allows to simulate the multi-physical behaviour of the HEMT and to observe the degradation in its structure. Then, we will define the CMA-ES method with its parameters and how to obtain the KA-CMA-ES method. In the next part, we will develop a coupling between the finite element model and the KA-CMA-ES model. Thanks to this coupling, we will solve the optimization problem. In the end, we will present the extracted results and compare the variation of the objective function before and after the optimization.

\section{High electron mobility transistor description}

The basic idea behind this component is to use as the conductive channel of a field-effect transistor, a twodimensional gas (2D-gas) of electrons circulating in a lightly doped material and resulting from the occupation of the energy levels of the potential well characteristic of a heterojunction [19]. Three electrodes commonly referred to as source, gate and drain to control the transistor operating current and voltage. The source and drain contacts are Ohmic type, the gate contact is Schottky type. For power amplification applications the common source circuit is used, the source electrode is connected to ground. The gate controls the current density flowing through the transistor by acting electrostatically on the electron gas, this is the low-power control electrode. The drain allows to control the operating voltage of the component, it is the highpower control electrode [20].

The HEMT is characterized by a particular structure (Fig. 1), Asif khan et al. have detailed the fabrication steps of HEMT and the creation of the heterojunction [21]. The structure of HEMT composed of several layers of different materials, one layer of silicon carbide (SiC) which represents the substrate on which the component is built. The nucleation layer is used to achieve a mesh tuning between the substrate and the GaN, GaN layer contains the 2DEG in its upper part, AlGaN is used to create a heterojunction with the GaN layer, there are other layers that perform other functions as Jacquet et al. have detailed in their paper $[22,23]$. In this work, the dimensions of the structure are $\mathrm{AlGaN}=0.03 \mu \mathrm{m}, \mathrm{GaN}=1.7 \mu \mathrm{m}$ and $\mathrm{SiC}=100 \mu \mathrm{m}$. And a gate width of $100 \mu \mathrm{m}$.

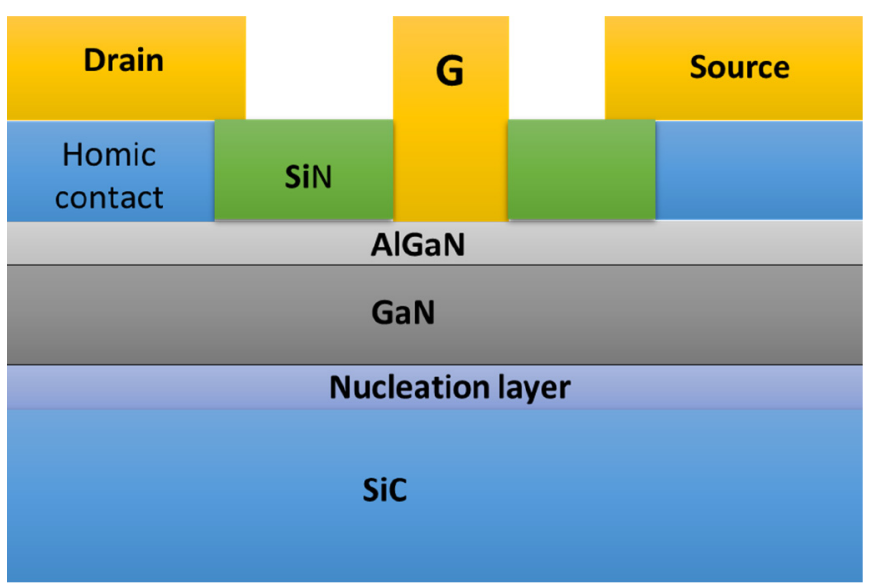

Fig. 1. HEMT structure.

\section{Electro-thermomechanical modeling of the HEMT}

In this part, we will present an electro-thermomechanical modeling study of the HEMT. We will start with the electro-thermal modeling and then thermomechanical modeling. The results of the numerical simulations will be presented.

\subsection{Electro-thermal modeling}

During HEMT's operation, it dissipates power at the active zone at the gate output [24], The dissipated power is a function of the voltage applied to the electrodes, and the current flowing through this transistor, such as [25]:

$$
P_{\text {diss }}=V_{d s} * I_{d s}
$$

where $V_{d s}$ and $I_{d s}$ are respectively the voltage and the current flowing between the drain and the source. To define the electrothermal behaviour of the transistor, we will inject the dissipated power at the level of the active zone of the HEMT. Thereafter, it is necessary to study the heat transfer at the component, this transfer is mainly by conduction, convection and radiation are negligible because they represent only less than $1.5 \%$ [26]. Based on this mode of heat exchange, the heat equation will be [27]:

$$
k . \nabla^{2} T=\rho . C_{p} \cdot \frac{\partial T}{\partial t}+Q
$$

where $Q$, power dissipated in joules; $K$, thermal conductivity $\left(\mathrm{W} . \mathrm{m}^{-1} \mathrm{~K}^{-1}\right) ; \rho$, density $\left(\mathrm{kg} . \mathrm{m}^{-3}\right) ; C_{p}$, thermal mass

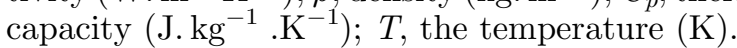

This equation allows us to describe the thermal behaviour of the transistor, as a function of the operating conditions and in particular the dissipated power [28]. The thermal properties required to develop the electrothermal model are listed in Table 1 , the thermal conductivity of some materials is a function of temperature $[16,29,30]$. 
Table 1. Materials thermal properties.

\begin{tabular}{llll}
\hline Materials & $\rho\left(\mathrm{Kg} \cdot \mathrm{m}^{-3}\right)$ & $K\left(W \cdot \mathrm{m}^{-1} \cdot K^{-1}\right)$ & $C_{p}\left(J \cdot K g^{-1} \cdot K^{-1}\right)$ \\
\hline $\mathrm{Au}$ & 19300 & 310 & 137 \\
$\mathrm{SiN}$ & 3300 & 10 & 713 \\
$\mathrm{AlGaN}$ & 5470 & $25 \cdot\left(\frac{293}{273+T}\right)^{L 35}$ & 548 \\
& 6100 & $161 \cdot\left(\frac{293}{273+T}\right)^{1.45}$ & 490 \\
$\mathrm{GaN}$ & 3220 & $416 \cdot\left(\frac{293}{273+T}\right)^{1.45}$ & \\
$\mathrm{SiC}$ & &
\end{tabular}

Table 2. Material's physical properties.

\begin{tabular}{llll}
\hline Materials & $E(\mathrm{GPa})$ & CTE $[1 / K]$ & Poisson's ratio \\
\hline $\mathrm{Au}$ & 70 & 14.2 & 0.44 \\
$\mathrm{SiN}$ & 250 & $1.5 \mathrm{e}-6$ & 0.27 \\
$\mathrm{AlGaN}$ & 212.75 & $5.2425 \mathrm{e}-6$ & 0.319 \\
$\mathrm{GaN}$ & 181 & $8.6 \mathrm{e}-6$ & 0.352 \\
$\mathrm{SiC}$ & 748 & $4.3 \mathrm{e}-6$ & 0.45 \\
\hline
\end{tabular}

\subsection{Thermomechanical modeling}

Thermomechanical modeling is used to study the influence of thermal behaviour on the mechanical behaviour of the HEMT structure. The distribution and the increase of the temperature in the HEMT due to the dissipated power can give rise to phenomena and mechanical degradations in the HEMT structure. To study these phenomena, we will develop a thermomechanical model, this model allows to link thermal parameters such as temperature to mechanical parameters such as stresses, strains and displacements. The coupling of the thermal model to the mechanical model can be translated into the form of equations, thanks to the thermoelasticity equations, strains and entropy density can be defined [27]:

$$
\begin{gathered}
\{\varepsilon\}=[D]^{-1}\{\sigma\}+\{\alpha\} \Delta T \\
\{S\}=\{\alpha\}^{T}\{\sigma\}+\frac{\rho \cdot C_{P}}{T_{0}} \Delta T ; \Delta T=T-T_{\text {ref }}
\end{gathered}
$$

where $\{\varepsilon\}$ : total strain vector $=\left[\varepsilon_{x} \varepsilon_{y} \varepsilon_{z} \varepsilon_{x y} \varepsilon_{y z} \varepsilon_{x z}\right]^{T}$; S: entropy density; $\{\sigma\}$ : stress vector; $T$ : ambient temperature; $T_{0}$ : absolute reference temperature; $T_{\text {ref: }}$ reference temperature; $\{\alpha\}$ : vector of thermal expansion coefficients; $\rho$ : volume density; $\mathrm{C}_{\mathrm{p}}$ : specific heat at constant pressure.

According to the thermodynamics law, the entropy density can be replaced by the heat density according to this equation:

$$
Q=T_{0} \cdot S
$$

The main equations become:

$$
\begin{gathered}
\{\varepsilon\}=[D]\{\varepsilon\}-\{\beta\} \Delta T \\
Q=T_{0}\{\beta\}^{T}\{\varepsilon\}+\rho \cdot C_{v} \cdot \Delta T
\end{gathered}
$$

where $\{\beta\}$ : thermoelastic coefficients vector $=[D]\{\alpha\} ; C_{v}$ : specific heat at constant volume $=C_{p}-\frac{T_{0}}{T}\{\alpha\}^{T}\{\beta\}$.

These two equations ensure the thermomechanical coupling, it allows to link the thermal parameters with the mechanical parameters. The two elementary couplings: electrothermal and thermomechanical ensure the global electro-thermomechanical coupling. The physical properties of the materials required to develop the model are listed in Table $2[31,32]$. Some properties are already integrated into the material library of Comsol multiphysics. 


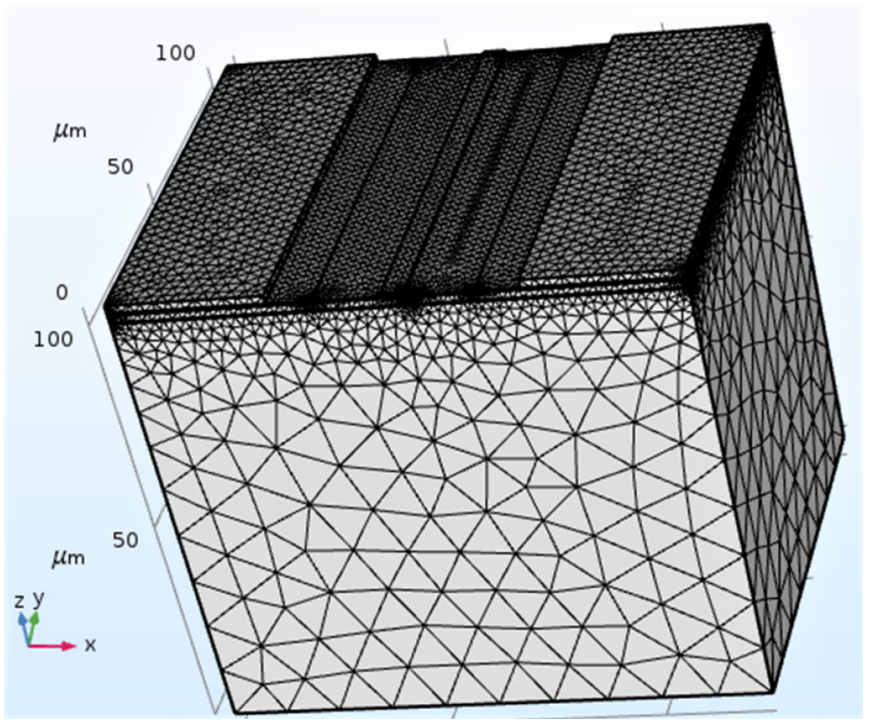

Fig. 2. The finite element model of the HEMT.

\subsection{Finite element model}

The HEMT electro-thermomechanical modeling is based on the resolution of the above coupling equations. The chosen method of solving is the finite element method (FEM) [33], it is the most used method for solving such problems, the analytical solution of the equations is very difficult. Therefore, it is necessary to introduce numerical methods. Comsol multiphysics software is based on the finite element method, it allows to reconstruct the system geometrically, to apply loads and boundary conditions and par to solve all equations related to the developed modeling (Figure 2). The recommended mesh in the case of HEMT modeling is the tetrahedral mesh, thanks to the good results obtained by this type. The mesh is very fine in the critical areas, and becomes normal in the rest of the component to optimize the calculation time.

\subsection{Evolution of the HEMT maximum temperature}

During operation, the power dissipated in the active area of the HEMT influences the thermal behaviour of the component. To observe the effect of the power dissipation on the transistor temperature, the linear density of this power is varied from $1 \mathrm{~W} / \mathrm{mm}$ up to $11 \mathrm{~W} / \mathrm{mm}$ keeping the other parameters constant, Figure 3 shows the variation of the HEMT temperature as a function of the dissipated power.

Figure 3 shows that the dissipated power influences the temperature, the impact results in a non-linear temperature evolution. The figure also presents a comparison of the simulation results and the experimental results [13]. We see that there is a good correlation between the two curves. Except for deviations in a few points, this deviation is because we have neglected other modes of heat transfer such as convection and radiation. We consider a good correlation as a sufficient indication to validate and consider our model for future simulations.

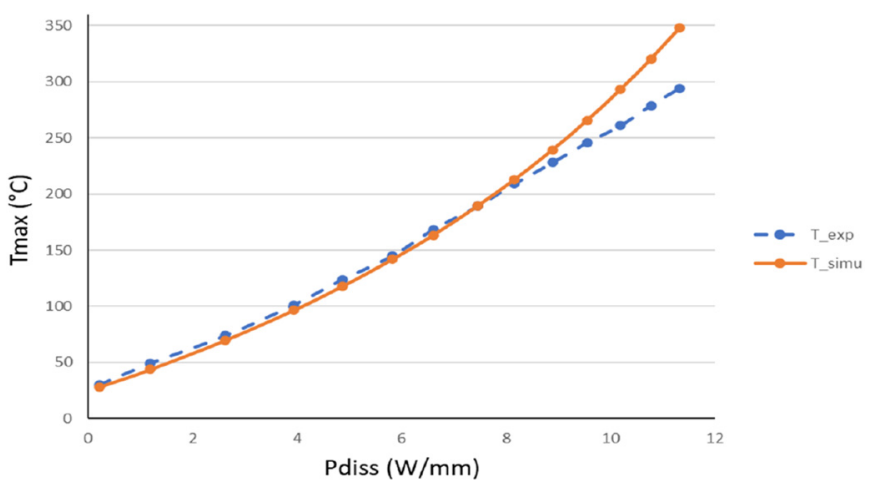

Fig. 3. Evolution of the HEMT maximum temperature.

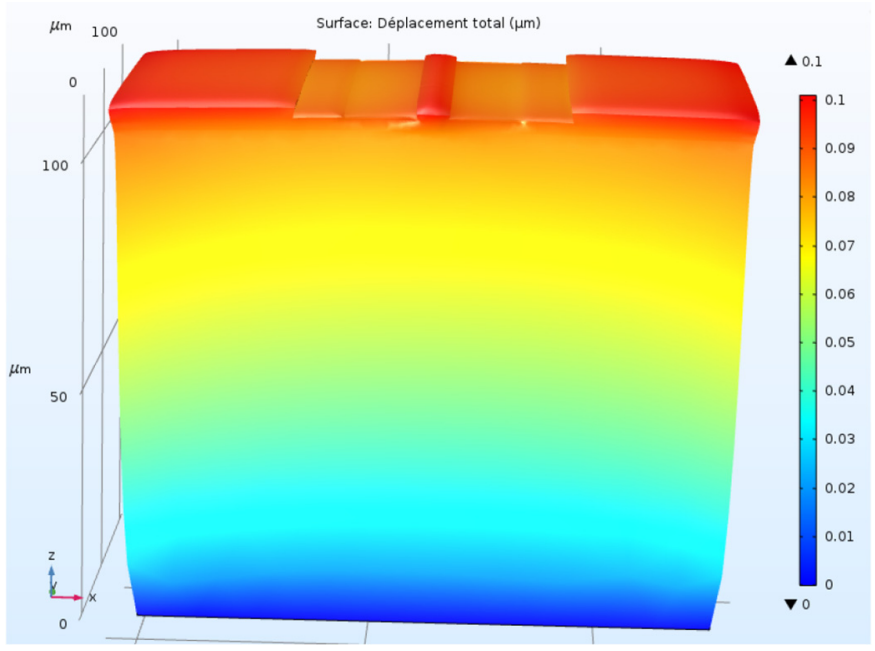

Fig. 4. Displacement distribution in the HEMT structure at $P_{\text {diss }}=9 \mathrm{w} / \mathrm{mm}$.

\subsection{Thermomechanical behaviour of the HEMT}

To study the mechanical behaviour of the HEMT, under operating conditions: the dissipated power and the reference temperature, the finite element model developed under Comsol multiphysics, allows to visualize the distribution of the mechanical parameters such as stresses, strains and displacements at the HEMT structure, as well as their evolutions according to the applied loads.

\subsubsection{Calculation of displacements in the HEMT structure}

The distribution of the displacements of the HEMT structure in Figure 4, is obtained from the 3D finite element model, according to the figure we can see that the displacements are very important at the electrodes of the HEMT, they expect a value of $0.1 \mu \mathrm{m}$ in the upper part of the structure. The operating conditions of the component have a great mechanical impact on the HEMT structure.

To observe the evolution of the displacement as a function of the applied loads, several simulations will be carried out in the different values of the dissipated power and a fixed reference temperature. The results are shown in Figure 5 , in this figure we can see that the displacements 


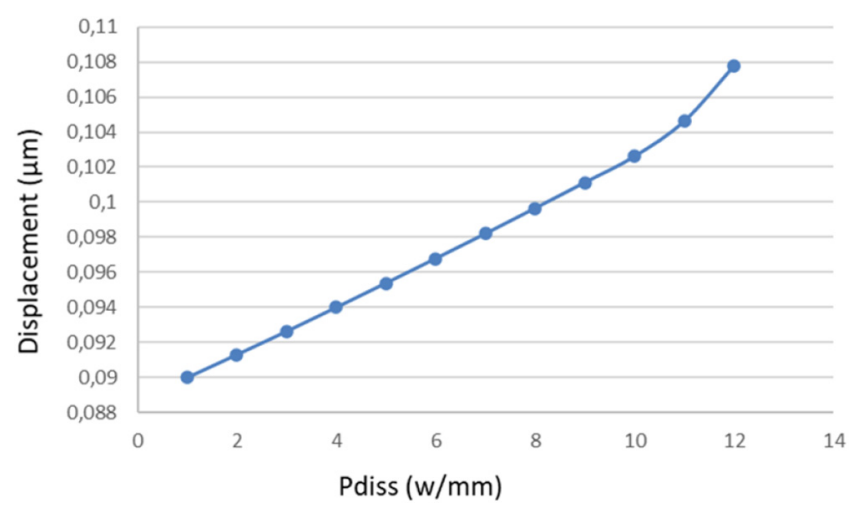

Fig. 5. Evolution of maximum displacements as a function of the power dissipated in the HEMT structure.

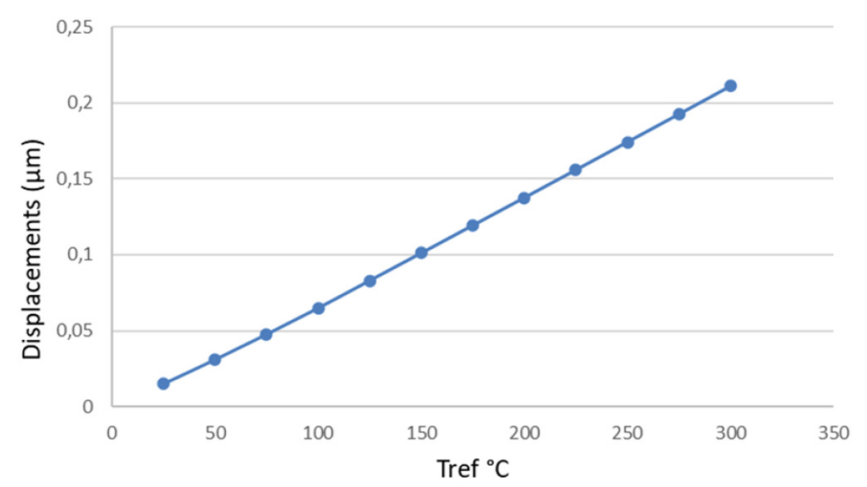

Fig. 6. Evolution of maximum displacements as a function of the reference temperature in the HEMT structure.

increase as a function of the dissipated power, following a non-linear evolution. At a dissipated power of $12 \mathrm{w} / \mathrm{mm}$, displacements attain $0.108 \mu \mathrm{m}$.

And to observe the influence of the reference temperature on the variation of the maximum displacements, we realized simulations for the different values of $T_{\text {ref }}$ from $25{ }^{\circ} \mathrm{C}$ to $300{ }^{\circ} \mathrm{C}$ and a fixed power dissipated. The results in Figure 6 shows that the maximum displacements increase as a function of the reference temperature, at $T_{r e f}=300^{\circ} \mathrm{C}$ displacements attain $0.21 \mu \mathrm{m}$. We also notice that $T_{\text {ref }}$ has a high impact than the power dissipated. Therefore, the operating conditions of the HEMT have a clear influence on the displacements in the structure of the HEMT.

\subsubsection{Calculation of strains in the HEMT structure}

The strain study is used to quantify how lengths have been expanded/contracted and angles have changed in a structure. To evaluate the strains in the HEMT structure, simulations are made using the finite element model developed previously. Figure 7 shows the distribution of the principal strains: the first and the second. The first strain is very high above and at the end of the gate. The second principal strain is higher than the first, it's very important over a large part of the gate. The operating
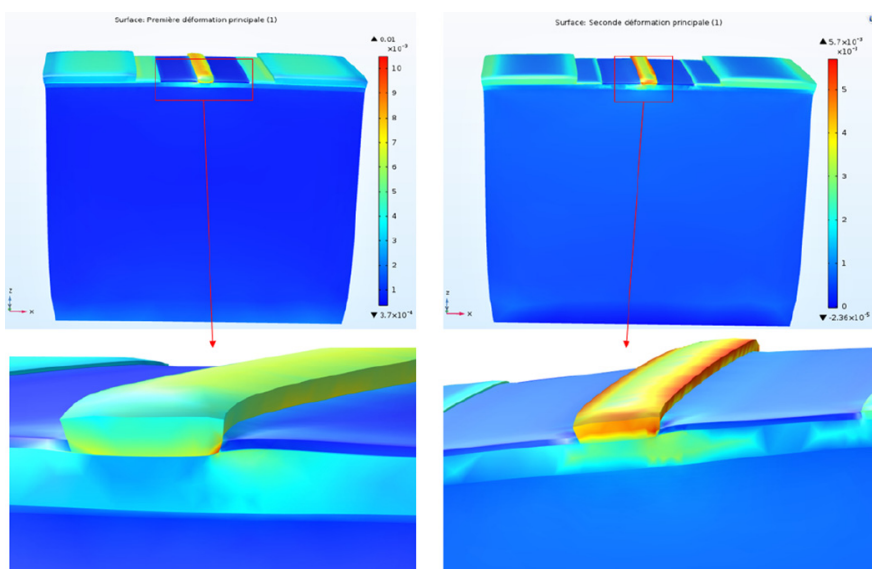

Fig. 7. Distribution of principal strains in the HEMT structure.
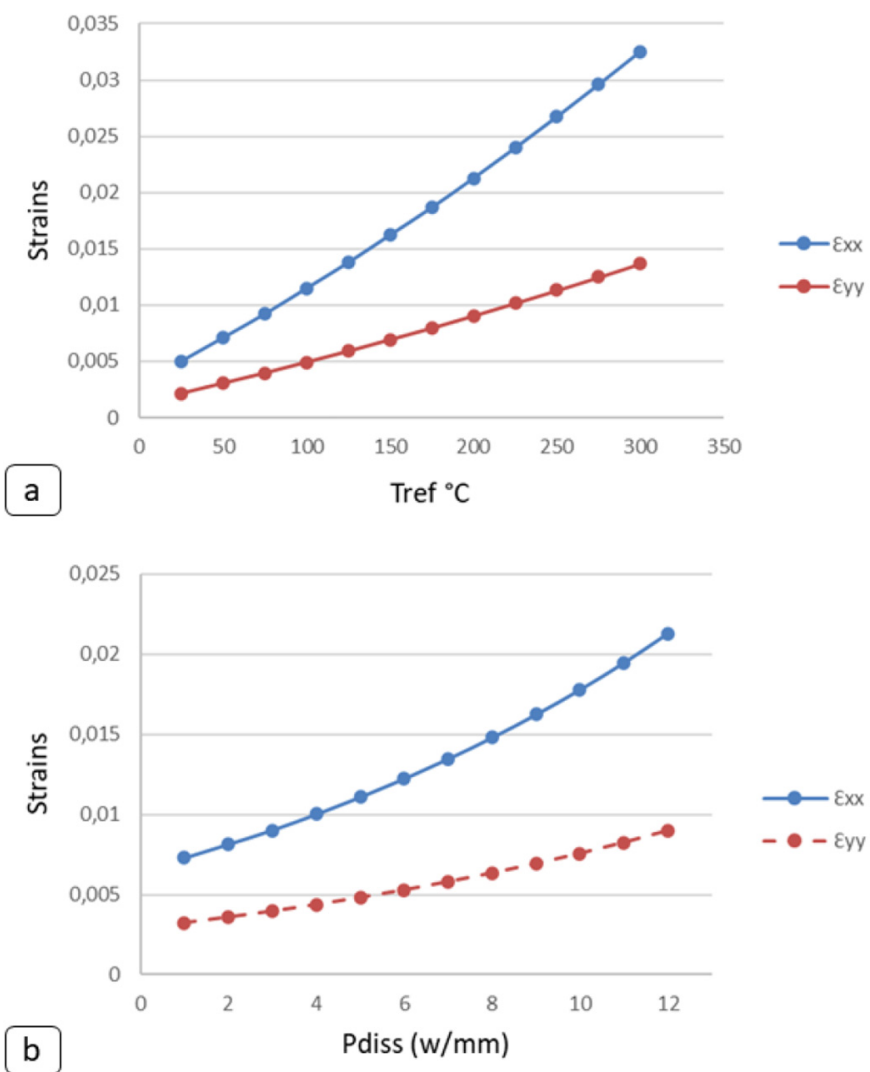

Fig. 8. Evolution of the maximum principal strains in the HEMT structure as a function of the power dissipated (a), and as a function of the reference temperature (b).

conditions generate strains in the HEMT structure, the maximum values are observed at the level of the gate as well. Knowing that the gate is an important layer in the structure and operation of the component, an increase in strain can lead to HEMT failure.

To observe the evolution of the strains as a function of the operating conditions, Figure 8 shows the evolution of 
the strains as a function of the power dissipated and the reference temperature. It is noticeable that the principal strains increase as a function of $P_{\text {diss }}$ and $T_{\text {ref }}$. The first principal strains are more important than the second. Moreover, the impact of the reference temperature is higher than that of the dissipated power.

\section{Optimization approach}

In this part, we will detail the optimization methods that we have used, starting with the definition of the CMA-ES method and its parameters. Then we will talk about the kriging metamodel, its management and quality control, and finally the global KA-CMA-ES method.

\subsection{Covariance matrix adaptation-evolution strategy (CMA-ES)}

The CMA-ES (Covariance Matrix Adaptation-Evolution Strategy) method is the best member of the family of evolution algorithms, it is a stochastic optimization method without derivatives and can adapt to non-convex optimization problems [34]. CMA-ES is based on the four principles of natural selection: evolution, selection, recombination and mutation [35]. The implementation of evolutionary operations ensures this approach, to guide the approach towards a global optimum in a space of continuous or discrete research [36]. CMA-ES $(\lambda, \mu)$ is based on the adaptation of the covariance matrix of the multinormal law in $R^{n}$, this adaptation is equivalent to the construction of an approximation of the objective function $\mathrm{f}$, this method generates $\lambda$ new individuals from $\mu$ elements of the population. CMA-ES $(\lambda, \mu)$ generates the population by sampling a multinormal distribution [35,37]:

$$
x_{k}^{(g+1)} \sim \boldsymbol{m}^{(g)}+\sigma^{(g)} \mathcal{N}\left(\boldsymbol{O}, \boldsymbol{C}^{(g)}\right)
$$

for $k=1, \ldots, \lambda$, where: $-\sim$ indicates the same distribution on the left and the right. $\mathrm{N}\left(\mathbf{0}, \boldsymbol{C}^{(g)}\right)$ is a multivariate normal distribution with a null mean and a covariance matrix $-\boldsymbol{C}^{(g)} ; \boldsymbol{x}_{k}^{(g+1)} \in \mathbb{R}^{n}, \quad k^{\text {th }}$ offspring (individual, research point) of the $g+1$ generation; $\boldsymbol{m}^{(g)} \in \mathbb{R}^{n}$, mean value of the distribution from research to generation $g$; $\sigma^{(g)} \in \mathbb{R}$, step size to generation $g ; C^{(g)} \in \mathbb{R}^{n \times n}$, covariance matrix at generation $g ; \lambda \geq 2$, the population size.

Then, the images of the individuals generated by sampling are evaluated by the objective function, the best individuals will be grouped in the mean vector $\mathrm{m}$, it has the expression:

$$
\boldsymbol{m}^{(g+1)}=\sum_{i=1}^{\mu} w_{i} \boldsymbol{x}_{i: \lambda}^{(g+1)}
$$

and

$$
\sum_{i=1}^{\mu} w_{i}=1, \quad w_{1} \geq w_{2} \geq \cdots \geq w_{\mu}>0
$$

where $\mu \leq \lambda$ the number of points selected or the size of the original population (parents); $w_{i=1 \ldots \mu} \in \mathbb{R}$, represents recombination weights, are strictly positive and verifying equation (10); $\mathbf{x}_{i: \lambda}$ : the $i$ th best individual selected from the set of individuals $\left[\mathbf{x}_{1}, \mathbf{x}_{2}, \ldots, \mathbf{x}_{\lambda}\right]$ generated by equation (9); $(i, \lambda)$ is the index of the $i$ th individual after ranking such that, $f\left(x_{1: \lambda}\right) \leq f\left(x_{2} \cdot \lambda\right) \leq \ldots \leq f\left(x_{\lambda: \lambda}\right)$ where $f$ is the objective function.

After updating the mean vector $\mathrm{m}$, the next step is the step adaptation $\sigma$ and the covariance matrix $\mathrm{C}$. The updating of the two parameters is done by two evolution paths $p_{c}$ and $p_{\sigma}$ [17]. however, the continuation of the CMA-ES method process requires to update the evolution path $p_{\sigma}$ allowing to update the step size $\sigma$, the two parameters are calculated according to the following equations:

$$
\begin{array}{r}
\mathbf{p}_{\sigma}^{(g+1)}=\left(1-c_{\sigma}\right) \mathbf{p}_{\sigma}^{(g)}+\sqrt{c_{\sigma}\left(2-c_{\sigma}\right)} \\
\sqrt{\mu_{w}}\left(\mathbf{C}^{(t)}\right)^{-\frac{1}{2}} \frac{\mathbf{m}^{(g+1)}-\mathbf{m}^{(g)}}{\sigma^{(t)}} \\
=\left(1-c_{\sigma}\right) \mathbf{p}_{\sigma}^{(g)} \\
+\sqrt{c_{\sigma}\left(2-c_{\sigma}\right)} \sqrt{\mu_{w}} \sum_{i=1}^{\mu} w_{i} \mathbf{z}_{i \lambda} \\
\sigma^{(g+1)}=\sigma^{(g)} \exp \left(\frac{c_{\sigma}}{d_{\sigma}}\left(\frac{\left\|\mathbf{p}_{\sigma}^{(g+1)}\right\|}{\left(\left\|\mathrm{E} \mathcal{N}_{d}(\mathbf{0}, \mathrm{I})\right\|\right)}-1\right)\right.
\end{array}
$$

$p_{\sigma}^{(g)} \in \mathbb{R}^{d}$ : the evolution path for the step size at generation $g$, its initial value is $\mathbf{p}_{\sigma}^{(0)}=0_{;} \in[0,1]$ : the time constant for step size adaptation, its default value is $c_{\sigma}=\left(\mu_{w}+2\right) /$ $\left(d+\mu_{w}+5\right) ; \mu_{w}$ : called effective selection mass variance, (effective selection mass variance), its value is $\mu_{w}=\left(\sum_{i=1}^{\mu} w_{i}\right)^{2} / \sum_{i=1}^{\mu} w_{i}^{2}$

To update the covariance matrix $C$, the evolution path $p_{c}$ must be updated, it is done according to the following equations:

$$
\begin{aligned}
& \mathbf{p}_{c}^{(g+1)}=\left(1-c_{c}\right) \mathbf{p}_{c}^{(g)}+h_{\sigma} \sqrt{c_{c}\left(2-c_{c}\right)} \sqrt{\mu_{u}} \frac{\mathbf{m}^{(g+1)}-\mathbf{m}^{(g)}}{\sigma^{(g)}} \\
& =\left(1-c_{c}\right) \mathbf{p}_{c}^{(g)}+h_{\sigma} \sqrt{c_{c}\left(2-c_{c}\right)} \sqrt{\mu_{w}} \sum_{i=1}^{\mu} w_{i}\left(\mathbf{C}^{(g)}\right)^{\frac{1}{2}} \mathbf{z}_{i: \lambda}
\end{aligned}
$$

whereas the covariance matrix becomes as follows:

$$
\begin{aligned}
C^{(g+1)}= & \left(1-c_{1}-c_{u}\right) \mathbf{C}^{(g)}+c_{1}\left(\mathbf{p}_{c}^{(g+1)}\left(\mathbf{p}_{c}^{(g+1)}\right)^{T}\right. \\
+ & \left.\delta\left(h_{\sigma}\right) \mathbf{C}^{(g)}\right) \\
& +c_{\mu} \sum_{i=1}^{u} w_{i}\left(\left(\mathbf{C}^{(g)}\right)^{\frac{1}{2}} \mathbf{z}_{i, \lambda}\right)\left(\left(\mathbf{C}^{(g)}\right)^{\frac{1}{2}} \mathbf{z}_{i: \lambda}\right)^{T}
\end{aligned}
$$


where $p_{c}^{(g)} \in \mathbb{R}^{d}$ : the evolution path for the step size at generation $g$, its initial value is $\mathbf{p}_{c}^{(0)}=0 ; c_{c} \in[0,1]$ : the adaptation constant of the covariance matrix; $h_{\sigma}$ : is the Heaviside function; $c_{1}$ and $c_{\mu}$ : expresses the learning rate for rank-one-update and rank- $\mu$-update, respectively. Their default values are $c_{1}=\frac{2}{(d+1.3)^{2}+\mu_{u v}}, \quad$ and $c_{u}=\min \left(1-c_{1}, 2 \frac{\mu_{v}-2+1 / \mu_{w}}{(d+2)^{2}+\alpha_{u} \mu_{w} / 2}\right)$ where $\alpha_{\mu}=2$, and $\delta\left(h_{\sigma}\right)=$ $\left(1-h_{\sigma}\right) c_{c}\left(2-c_{c}\right)$.

All these steps can be organized in an algorithm, which runs according to the chosen convergence conditions, following a very precise order, the algorithm repeats itself until the convergence condition is satisfied by returning the best individuals.

\subsection{Kriging metamodel}

During the development and design of complex equipment and systems, detailed modeling is required to evaluate and improve these systems in the development process. Modeling is done by several methods such as the finite element method, it's based on detailed simulations that usually take a long time to run. And sometimes it is necessary to perform hundreds or thousands of simulations, as in the case of reliability or optimization studies to obtain good results, which is impractical in terms of time. This constraint leads us to look for other relevant methods. However, metamodels are simplified models of detailed simulation models. Evaluations using metamodels are more efficient and more feasible compared to detailed models in terms of time. Among the most commonly used types of metamodels is the kriging metamodel. The kriging model is Gaussian process-based modeling to interpolate deterministic data without noise. The kriging model is given in form [38]:

$$
Y(x)=m(x)+Z(x) .
$$

It has two components: $\mathrm{m}(\mathrm{x})$ is a deterministic function or global trend function, and the Gaussian random function $Z(x)$ which is a stationary Gaussian process, i.e., with constant mean. It is often assumed to be centred: $E(Z(x))=0$ for all $x$ and whose covariance between two points is only dependent on their distance:

$$
\operatorname{cov}\left(Z(x), Z\left(x^{\prime}\right)\right)=\sigma^{2} r\left(x-x^{\prime}\right)
$$

$\operatorname{cov}\left(Z(x), Z\left(x^{\prime}\right)\right)$ is the covariance of $\mathrm{Z}(\mathrm{x})$ between two points $\mathrm{x}$ and $\mathrm{x}$ '. $\sigma$ is the variance of the Gaussian process and, $\mathrm{r}$ is the correlation function, there are several correlation functions but, the most used is the Gaussian correlation function, it is expressed by:

$$
R\left(\mathbf{x}^{(i)}, \mathbf{x}^{(j)}\right)=\exp \left[-\sum_{k=1}^{D} \theta_{k}\left|x_{k}^{(i)}-x_{k}^{(j)}\right|^{2}\right]
$$

where $x_{k}^{(i)}$ and $x_{k}^{(j)}$ are respectively the $k^{(t h)}$ component of the sampling point $x^{(i)}$ and $x^{(j)}, \mathrm{D}$ is the dimension of the design space, and $\theta_{k}(k=1,2,3, \ldots, D)$ are the unknown parameters of the correlation function. The mean and variance of the prediction for any untested point $\mathrm{x}$ can be given respectively by:

$$
\begin{gathered}
\hat{y}(\mathrm{x})=\beta+\mathbf{x}^{\mathrm{T}}(\mathbf{x}) \mathbf{R}^{-1}\left(\mathrm{y}_{\mathrm{s}}-\beta \mathbf{I}\right) \\
\mathrm{s}^{2}(\mathrm{x})=\sigma^{2}\left[\mathbf{1}-\mathrm{r}^{\mathrm{T}}(\mathrm{x}) \operatorname{Rr}(\mathrm{x})+\frac{\left(1-\mathrm{I}^{\mathrm{T}} R-1 \mathrm{r}(\mathrm{x})\right)^{2}}{\mathrm{I}^{\mathrm{T}} \mathrm{R}^{-1} \mathrm{I}}\right]
\end{gathered}
$$

where $-y(\mathbf{x})$ is the estimated mean value of $\mathrm{y}(\mathrm{x}) ; s^{2}(\mathbf{x})$ is the estimated variance of $\mathrm{y}^{\wedge}(\mathrm{x}) ; r(\mathrm{x})$ is the vector of correlation functions between the untested point $\mathrm{x} ; y_{\mathrm{s}}$ is the vector of observed responses corresponding to the sampled set; $I$ is a unit vector and the $\mathrm{N}$ samples $\left(x_{1} \ldots \ldots x_{N}\right)$, i.e., $r(x)=\left[R\left(x, x_{1}\right), \ldots, R\left(x, x_{N}\right)\right]^{T}, \mathrm{R}$ is the $\mathrm{N} \times \mathrm{N}$ matrix of correlation functions for the sampled data. The estimation of the unknown parameters can be performed using the maximum likelihood estimation method [39].

\subsection{Metamodel management}

When using the metamodel to predict the original function, two criteria must be satisfied. The first is that the evolutionary algorithm must converge towards the global optimum. The second is the cost of the computation, it must be reduced as much as possible. the main constraint to replace the original function by a metamodel is to build a very precise metamodel, which allows to efficiently predict the original function. Because there is the risk of converging towards a false optimum. To avoid this problem, the original function and the metamodel must be used together. The original function is used to evaluate all the individuals of a generation or some of the individuals. Every individual evaluated by the original function is called a controlled individual. Similarly, a generation in which all its individuals are evaluated using the original function is called a controlled generation [39,40]. Metamodel management is generally divided into three principal approaches from an evolution control point of view:

- Uncontrolled evolution: consists in assuming that the metamodel is of high quality. Therefore, the original function of the evaluation is not used in the evolution calculation, i.e., no individual or generation is not controlled.

- Evolution with fixed control: in this case, the control frequency is fixed. There are two approaches to fixed control, the first is evolution control based on individuals, the second is evolution control based on generation.

- Evolution with adaptive control: this approach considers that evolution control depends on the quality of the metamodel, individuals are controlled iteratively to update the metamodel until its quality is acceptable.

\subsection{Metamodel quality}

The quality of the metamodel is a very important issue for its use in evolutionary computation, good metamodel quality ensures a correct evaluation of individuals by the original function, therefore a correct selection of 
individuals. In the case of evolutionary computation, the metamodel must allow the evolutionary algorithm to select the best individual. CMA-ES uses a selection based on ranking, for each iteration, it evaluates the original function for each individual of the generation. The metamodel quality is based on its ability to correctly classify individuals in the population. However, the approximate classification procedure is an efficient method to control the quality of the metamodel without knowing the exact classification of the complete generation [40]. The approximate classification procedure proposed by Huang et al. [39] is composed of several steps classified in the form of an algorithm (Algorithm 1). In this algorithm he has made two modifications to the process: the first is instead of selecting a single individual for reassessment and updating the metamodel, he only selects $n_{\text {init }}=\max (1,0.3 \lambda)$ individuals, where $\lambda$ is the size of the population. Second, it uses a lot size $\mathrm{nb}=\max (1$, $[\lambda / 10])$ which is proportional to $\lambda$, in the iteration loop of the approximate ranking procedure [39].

\subsection{Kriging assisted CMA-ES}

To perform an optimization study using the CMA-ES method, it is necessary to realize several (thousands) simulations using the finite element model, and each simulation can last tens of minutes, which is costly in terms of time. However, the integration of the kriging metamodel into the CMA-ES algorithm has been considered using the approximate ranking procedure as a quality control method for the metamodel. The CMA-ES algorithm will keep all its main instructions, except that the step of evaluation of the image of individuals, it can be by the original function or by the metamodel. In the second case, it is done by calling the approximate ranking procedure if $n_{k}$ the number of points required to build the metamodel is greater than the number of points $\mathrm{T}$ in the training set. This integration of the metamodel in the evolutionary method can take the form of an algorithm named KA-CMA-ES (Algorithm 2) [39,40].

\section{Results and discussion}

\subsection{Optimization problem}

The modeling of the HEMT has shown that the operating conditions such as the dissipated power and the reference temperature generate degradations in HEMT structure, these degradations have several forms: displacements, strains, stresses and others. These parameters affect the reliability and performance of the component, especially when they reach critical values. Stress is an elementary component of cohesion efforts or an elementary effort applied to an elementary surface. Stress is a force divided by a surface. When the load is applied, the stress can often no more be defined as Normal or Tangential. A stress equivalent to normal stress is then useful to carry out a degradation study. These equivalent stresses are most

\section{Algorithm 1 Approximate ranking procedure}

- given: $\quad\left(z_{k}, x_{k}\right)_{k-1}^{\lambda} \cdot m^{(g)}, \sigma^{(s)}, C^{(g)}, t, \mathcal{A}, f(x)$ - approximate: build surrogate model $f$ based on $\mathcal{A}$, and predict $f_{k}=f\left(\mathbf{x}_{k}\right), k=1, \cdots, \lambda$

- rank and determine the parent set $\mathcal{P}_{1}=\left\{\mathbf{x}_{i: \lambda}\right\}_{i=1}^{j x}$ where $\hat{f}\left(\mathbf{x}_{1}: \lambda\right) \leq \cdots \leq \hat{f}\left(\mathbf{x}_{2: \lambda}\right)$

- select $n_{\text {init }}$ controlled individual based on criterion $C\left(\mathbf{x}_{k}\right), k=1, \cdots, \lambda$ - evaluate the $n_{\text {init }}$ selected individuals by $f(\mathbf{x})$ and update set $\mathcal{A}, t \leftarrow t+n$ init-

- for $m=2:\left(\lambda-n_{\text {init }}\right) / m_{b}$ do

- approximate: build model $\hat{f}$ based on $\mathcal{A}$, and predict $f_{k}=\hat{f}\left(\mathbf{x}_{k}\right), k=1, \cdots, \lambda$

- rank and determine the parent set $\left.\mathcal{P}_{m}=\mid \mathbf{x}_{i: \lambda}\right\}_{i=1}^{\mu}$ where $\hat{f}\left(\mathbf{x}_{1: \lambda}\right) \leq \cdots \leq \hat{f}\left(\mathbf{x}_{\lambda: \lambda}\right)$

- if $P_{m-1} \neq \mathcal{P}_{\mathrm{m}}$ (the parent set has changed) then select $n_{b} \quad$ controlled individual based

criterion

$C\left(\mathbf{x}_{k}\right), k=1, \cdots, \lambda$ andx $_{k} \notin \mathcal{A}$

- evaluate the $n_{b}$ selected individuals by $f(\mathbf{x})$ and update set

$\mathcal{A}, t<t+n_{b}$

- else

break (exit for loop)

- end if

- end for

- output: $t, \mathcal{A},\left(\mathbf{x}_{k}, \mathbf{z}_{k}, f_{k}\right)_{k}^{\lambda}-1$

often defined according to Tresca or von Mises criteria. In this sense, the von Mises criterion is the most used, it is based on the principal stresses [41]:

$$
\sigma_{e}=\frac{1}{\sqrt{2}} \sqrt{\left(\sigma_{1}-\sigma_{2}\right)^{2}+\left(\sigma_{2}-\sigma_{3}\right)^{2}+\left(\sigma_{3}-\sigma_{1}\right)^{2}}
$$

where $\sigma_{e}$ is the equivalent von Mises constraint, $\sigma_{1}, \sigma_{2}$ and $\sigma_{3}$ are respectively the first, second and third principal constraint. This criterion allows us to identify the elements of the structure which have a great tendency to degrade (rupture). Figure 9 shows the distribution of von Mises constraints in the HEMT structure. In this case, as the load $P_{\text {diss }}=9 \mathrm{w} / \mathrm{mm}$ and $T_{\text {ref }}=150{ }^{\circ} \mathrm{C}$, the von Mises stresses are important in the lower part of the substrate because this layer receives the reference temperature from the external environment and is also the basis of the structure construction. The stresses are also important at the AlGaN layer in both sides of the gate, this layer is very close to the active zone where the power is dissipated. It is also remarkable that the structure undergoes deformations at the several layers beside the active zone, especially the AlGaN layer and the passivation layer and the gate too. All these degradation phenomenons can influence HEMT reliability.

To optimize the reliability of the HEMT, the operating conditions cannot be controlled, as they are imposed. On the other hand geometrical parameters influence the mechanical behavior of the structure, these parameters 
Algorithm 2. Kriging-assisted CMA-ES.

Initialize evolution path $p_{s}^{(0)}=0, p_{c}^{(0)}=0$, the covariance matrix $C^{(i)}=I$ the step size $a^{\text {ion }}$ and the selection parameters. Initialize the mean vector $m^{(0)}$ to a random candidate $g-0$ while Convergence criterion is not renched do

$T \leftarrow\left\{\left(x_{i}, f_{i}\right) \in \mathcal{S} \|\left(x_{i}-m^{(b)}\right)^{T}\right.$

$\left(\left((\infty)^{2} c^{4}\right)-1\left(x_{i}-m^{(y)}\right) \leq r^{2}\right\}$

if $|T|<m_{2}$ then

evaluation

$f_{k}-f\left(x_{k}^{(s+1)}\right) k=1, \ldots, \lambda$

$\mathcal{S}=\mathcal{S} \cup\left\{\left(x_{k}^{\langle 9+1)}, f_{k}\right)\right\}_{\varepsilon-1}^{\lambda}$

$\leftarrow\left\{\left(x_{i}, f_{i}\right) \in \mid\left(x_{i}-m^{(g)}\right)^{T}\left(\left(\sigma^{(g)}\right)^{2} C^{(\Delta)}\right)^{-1}\left(x_{i}-m^{\langle g]}\right) \leq r^{2}\right\}$

else

$\hookrightarrow$ Metamodel-based evaluation

Run approximate ranking according to Algorithm 1 .

end if

$$
\begin{aligned}
& m^{(g+1)}=\sum_{i=1}^{\mu} w_{i} x_{i=\lambda}^{(s)} \\
& p_{\sigma}^{(g+1)}=\left(1-c_{\sigma}\right) p_{\sigma}^{(g)}+\sqrt{c_{\sigma}\left(2-c_{\sigma}\right) \mu_{e f f}}\left(C^{(g)}\right)^{-\frac{1}{2}} \frac{m^{(g+1)}-m^{(g)}}{\sigma^{-(g)}} \\
& \sigma^{(g+1)}=\sigma^{(g)} \exp \left(\frac{c_{\sigma}}{d_{\sigma}}\left(\frac{\left\|p_{\sigma}^{(g+1)}\right\|}{E(\| N(0, I) \mid}-1\right)\right) \\
& p_{c}^{(g+1)}=\left(1-c_{c}\right) p_{c}^{(g)}+h_{\sigma} \sqrt{c_{c}\left(2-c_{c}\right) \mu_{e f f}} \frac{m^{(g+1)}-m^{(g)}}{\sigma^{(g)}} \\
& C^{(g+1)}=\left(1-c_{1}-c_{u}+c_{1} \delta\left(h_{\sigma}\right)\right) C^{(g)}+c_{1} p_{c}^{(g+1)}\left(p_{c}^{(g+1)}\right)^{T} \ldots \\
& +c_{\mu} \sum_{i=1}^{u} w_{i}\left(\frac{x_{i: \lambda}^{(g+1)}-m^{(g)}}{\sigma^{(g)}}\right)\left(\frac{x_{i=1}^{(g+1)}-m^{(g)}}{\sigma^{(g)}}\right)^{T} \\
& \text { end while } \\
& \text { return best optimum } x_{\mathrm{opt}}
\end{aligned}
$$

can be variables to determine the best dimensions of the structure that ensure the good reliability of the structure. Therefore, the optimization problem is to minimize the von Mises stresses under geometrical constraints:

$$
\left\{\begin{array}{l}
\min (f(x))=\min \left(\sigma_{e}\right) \\
100<a<160 \\
0.1<b<1 \\
1.1<c<1.9
\end{array}\right.
$$

where $a, b$ and $c$ are respectively substrate thickness, nucleation thickness and GaN thickness. To solve the optimization problem, the coupling between two models has been developed: the finite element model developed under Comsol multiphysics software, and the statistical model of KA-CMA-ES coded under Matlab software. The statistical model is used to determine the optimal values that minimize the objective function and to build the metamodel necessary for the calculation. The finite element model makes it possible to compute the values of the von Mises stresses for each variation of the geometrical parameters.

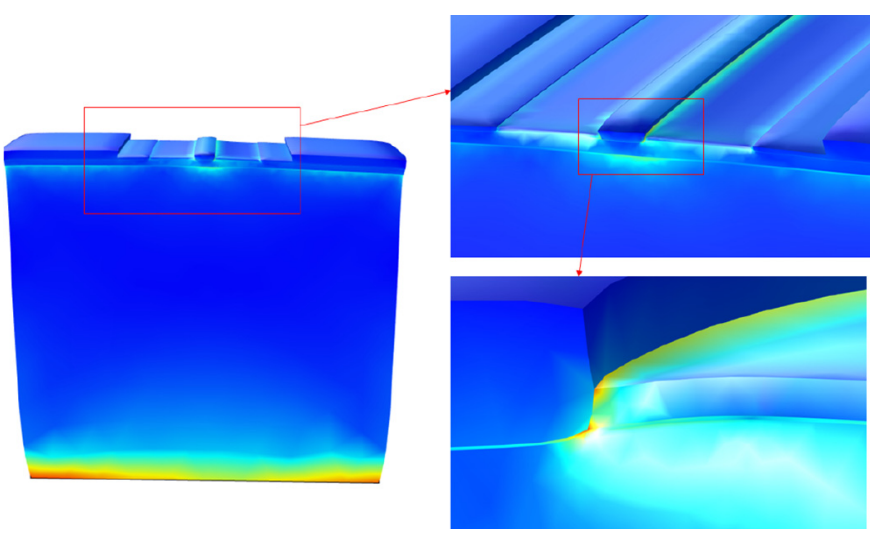

Fig. 9. Distribution of von Mises stresses in the HEMT structure. the red color indicates the areas with maximum stresses, and the blue color indicates the minimum values.

\subsection{Optimization results}

After the application of optimization processes, the results obtained are classified in Table 3.

The optimization results in Table 3 are simulation results in power dissipation of $9 \mathrm{w} / \mathrm{mm}$ and a reference temperature of $300^{\circ} \mathrm{C}$. The CMA-ES and KA-CMA-ES optimization approaches that we have developed have allowed us to determine the optimal dimensions, which minimize the maximum von Mises stresses. This reduces the probability of failure in the structure and improves its reliability. The KA-CMA-ES method is more efficient in terms of the calculation time $(t)$ and the number of simulations $(\mathrm{N})$ required to reach the best optimum. To observe the variation of the von Mises stresses before and after the optimization process, simulations are made in both cases, the results are presented in Figure 10.

\section{Conclusion}

In this work, the electro-thermomechanical modeling of the high electron mobility transistor was performed. This modeling allowed to describe the thermal and mechanical behaviour as a function of the operating conditions. The finite element model is developed under the Comsol multiphysics software. After applying the loads to the model, the maximum operating temperature of the HEMT increases in a non-linear evolution as a function of the dissipated power. Also, degradation phenomena like displacements and deformations in the transistor structure have been observed. Therefore, we developed an optimization approach to reduce the effect of these degradations. In this case, we took only three geometric parameters, this method is based on the integration of the CMA-ES method with the kriging metamodel. After applying this approach, von Mises constraints in the HEMT structure were minimized. CMA-ES with kriging showed efficiency in solving optimization problems. 
Table 3. Optimization results using CMA-ES and KA-CMA-ES.

\begin{tabular}{llll}
\hline Parameters & Initials values & CMA-ES & KA-CMA-ES \\
\hline$a$ & 100 & 149.32 & 148.48 \\
$b$ & 0.3 & 0.61 & 0.63 \\
$c$ & 1.7 & 1.34 & 1.34 \\
$N$ & - & 8316 & 6039 \\
$t(s)$ & - & 208926 & 182360 \\
\hline
\end{tabular}

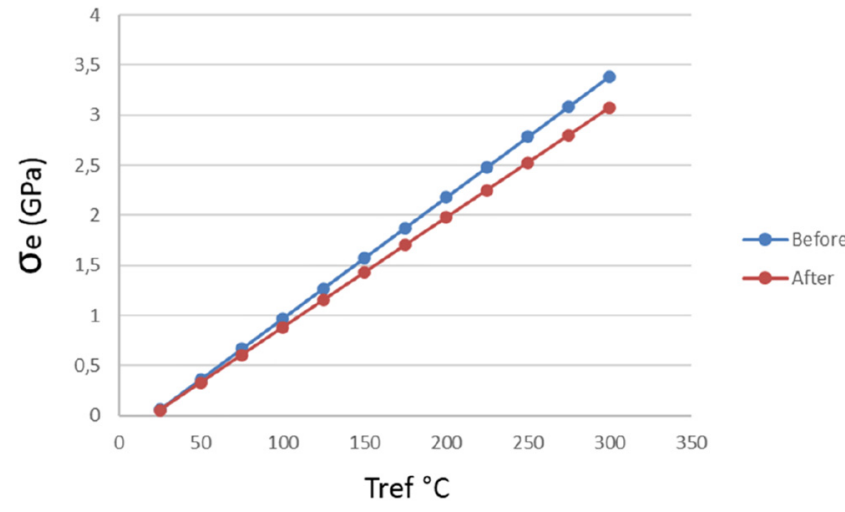

Fig. 10. Evolution of von Mises constraints before and after the optimization process.

\section{References}

1. S.S. Sajjadi Jaghargh, A.A. Orouji, An AlGaN/GaN HEMT by a reversed pyramidal channel layer: Investigation and fundamental physics, Int. J. Numer. Modell. 13-18 (2020)

2. T. Mimura, S. Hiyamizu, T. Fujii, K. Nanbu, A new fieldeffect transistor with selectively doped gaas/n-alxga1-xas heterojunctions, Jpn. J. Appl. Phys. 19, L225 (1980)

3. S. García, I. Ñiguez-De-La-Torre, J. Mateos, T. González, S. Pérez, Impact of substrate and thermal boundary resistance on the performance of AlGaN/GaN HEMTs analyzed by means of electro-thermal Monte Carlo simulations, Semiconduct. Sci. Technol. 31 (2016)

4. L. Wang, J. Liu, W. Zhou, Z. Xu, Y. Wu, H. Tao, A novel method to dynamic thermal impedance and channel temperature extraction of GaN HEMTs, Int. J. Numer. Modell. 1-9 (2019)

5. D.Y. Jeon, D.K. Kim, S.J. Park, Y. Koh, C.Y. Cho, G.T. Kim, K.H. Park, Effects of series resistance and interface properties on the operation of $\mathrm{AlGaN} / \mathrm{GaN}$ high electron mobility transistors, Microelectr. Eng. 199, 40-44 (2018)

6. Y. Dong, Z. Xie, D. Chen, H. Lu, R. Zhang, Y. Zheng, Effects of dissipative substrate on the performances of enhancement mode AlInN/GaN HEMTs, Int. J. Numer. Modell. Electr. Netw. Dev. Fields 32, 1-9 (2019)

7. Y. Chen, Y. Xu, F. Wang, C. Wang, Y. Zhang, B. Yan, R. Xu, Improved quasi-physical zone division model with analytical electrothermal Ids model for AlGaN/GaN heterojunction high electron mobility transistors, Int. J. Numer. Modell. Electr. Netw. Dev. Fields 1-17 (2019)
8. J. Das, H. Oprins, H. Ji, A. Sarua, W. Ruythooren, J. Derluyn, M. Germain, G. Borghs, A temperature analysis of high-power algan/gan hemts, Dans Proceedings of 12 th International Workshop on Thermal investigations of ICs (2006) 2-5

9. Y. Jia, Y. Xu, Y. Guo, A universal scalable thermal resistance model for compact large-signal model of AlGaN/GaN HEMTs, IEEE Trans. Microw. Theory Tech. 66, 4419-4429 (2018)

10. H. Belmabrouk, B. Chouchen, E.M. Feddi, F. Dujardin, I. Tlili, M. Ben Ayed, M.H. Gazzah, Modeling the simultaneous effects of thermal and polarization in InGaN/GaN based high electron mobility transistors, Optik 163883 (2019)

11. U. Radhakrishna, Physics-Based III-Nitride Device Modeling (Elsevier Inc., 2019), 1st edn. Vol. 102

12. S. Shamsir, F. Garcia, S.K. Islam, Modeling of enhancementmode gan-git for high-power and high-temperature application, IEEE Trans. Electr Dev. 67, 588-594 (2020)

13. Fully coupled thermoelectromechanical analysis of GaN high electron mobility transistor degradation, J. Appl. Phys. 111, 0-16 (2012)

14. J.P. Jones, M.R. Rosenberger, W.P. King, R. Vetury, E. Heller, D. Dorsey, S. Graham, Electro-thermo-mechanical transient modeling of stress development in algan/gan high electron mobility transistors (hemts) (2014) 959-965

15. M.A. Alim, S. Afrin, A. Rezazadeh, C. Gaquiere, Thermal response and correlation between mobility and kink effect in gan hemts, Microelectr. Eng. (2020)

16. L. Baczkowski, J.-C. Jacquet, O. Jardel, C. Gaquière, M. Moreau, D. Carisetti, L. Brunel, F. Vouzelaud, Temperature measurements in rf operating conditions of algan/gan hemts using ir microscopy and raman spectroscopy (2015) 152-155

17. N. Hansen, The CMA evolution strategy: a tutorial. Preprint arXiv:1604.00772v1 (2104)

18. A. Makhloufi, Y. Aoues, A. El Hami, B. Radi, P. Pougnet, D. Delaux, Study on the thermomechanical fatigue of electronic power modules for traction applications in electric and hybrid vehicles (IGBT), in: Reliability of High-Power Mechatronic Systems (Elsevier, 2017), pp. 213-251

19. R. Aubry, J.-C. Jacquet, J. Weaver, O. Durand, P. Dobson, G. Mills, M.-A. di Forte-Poisson, S. Cassette, S.-L. Delage, Sthm temperature mapping and nonlinear thermal resistance evolution with bias on algan/gan hemt devices, IEEE Trans. Electr. Dev. 54, 385-390 (2007)

20. A. Amar, B. Radi, A. El Hami, La modélisation thermique de transistor a haute puissance de type HEMT, Incertitudes et fiabilité des systèmes multiphysiques 3, 1-7 (2019)

21. M. Asif Khan, A. Bhattarai, J. Kuznia, D. Olson, High electron mobility transistor based on a gan-al $\mathrm{x}$ ga1- $\mathrm{x} \mathrm{n}$ heterojunction, Appl. Phys. Lett. 63, 1214-1215 (1993) 
22. I. Nifa, C. Leroux, A. Torres, M. Charles, D. Blachier, G. Reimbold, G. Ghibaudo, E. Bano, Characterization of 2DEG in $\mathrm{AlGaN} / \mathrm{GaN}$ heterostructure by Hall effect, Microelectr. Eng. 178, 128-131 (2017)

23. D.C. Streit, K.L. Tan, P.-H. Liu, High power pseudomorphic gallium arsenide high electron mobility transistors, uS Patent 5, 262, 660 (Nov. 16 1993)

24. A.A. Wilson, N.R. Jankowski, F. Nouketcha, R. Tompkins, Kapitza resistance at the two-dimensional electron gas interface 766-771 (2019)

25. L. Baczkowski, Modélisation et Caractérisation Thermique de Transistors de Puissance Hyperfréquence GaN et Conséquences sur la Fiabilité de Modules Radars d'Émission/Réception en Bande X, Ph.D. thesis, Université de Lille (2015)

26. R. Aubry, Etude des aspects électrothermiques de la filière hemt algan/gan pour application de puissance hyperfréquence, Ph.D. thesis, Lille 1 (2004)

27. A. El Hami, P. Pougnet, Embedded Mechatronic Systems 2: Analysis of Failures, Modeling, Simulation and Optimization, ISTE éditions, 2015

28. COMSOL Multiphysics modeling software. URL https:// www.comsol.fr/

29. S. Cheng, P.C. Chou, Novel packaging design for high-power GaN-on-Si high electron mobility transistors (HEMTs), Int. J. Thermal Sci. 66, 63-70 (2013)

30. O. Jardel, J.-C. Jacquet, L. Baczkowski, D. Carisetti, D. Lancereau, M. Olivier, R. Aubry, M.-A. di Forte Poisson, C. Dua, S. Piotrowicz, S.L. Delage, InAlN/GaN HEMTs based L-band high-power packaged amplifiers, Int. J. Microw. Wireless Technolog. 6, 565-572 (2014)
31. L.E. Stevens, Thermo-piezo-electro-mechanical simulation of algan (aluminum gallium nitride)/gan (gallium nitride) high electron mobility transistor, Ph.D. thesis (2013)

32. M.A.D. Maur, A.D. Carlo, AlGaN/GaN HEMT degradation : an electro-thermo-mechanical simulation, IEEE Trans. Electr. Dev. 60, 3142-3148 (2013)

33. T.M. Wagner, A very short introduction to the Finite Element Method (1908) (2004) 1-14.

34. H. Smaoui, L. Zouhri, S. Kaidi, E. Carlier, Combination of fem and cma-es algorithm for transmissivity identification in aquifer systems, Hydrolog. Process. 32, 264-277 (2018)

35. O. Sigaud, F. Stulp, Adaptation de la matrice de covariance pour l'apprentissage par renforcement direct., Rev. d'intelligence Artif. 27, 243-263 (2013)

36. H. Hamdani, B. Radi, A. El Hami, Optimization of solder joints in embedded mechatronic systems via Kriging-assisted CMA-ES algorithm, Int. J. Simul. Multidiscipl. Des. Optim. 10, A3 (2019)

37. O.A. Elhara, Stochastic black-box optimization and benchmarking in large dimensions (2017)

38. A. El Hami, R. Bouchaib, Uncertainty and optimization in structural mechanics, Wiley Online Library (2013)

39. C. Huang, B. Radi, A. El Hami, H. Bai, CMA evolution strategy assisted by kriging model and approximate ranking, Appl. Intell. 48, 4288-4304 (2018)

40. H. Hamdani, B. Radi, A. El Hami, Metamodel assisted evolution strategies for global optimization of solder joints reliability in embedded mechatronic devices, Microsyst. Technolog. 25, 3801-3812 (2019)

41. Desjuzeur, Résistance des matériaux, Houille Blanche c 134-137 (1912)

Cite this article as: Abdelhamid Amar, Bouchaïb Radi, Abdelkhalak El Hami, Optimization based on electro-thermo-mechanical modeling of the high electron mobility transistor (HEMT), Int. J. Simul. Multidisci. Des. Optim. 13, 2 (2022) 